\title{
Reviewers Acknowledgements
}

Journal of Academic Finance wishes to acknowledge the following individuals for their assistance with peer review of manuscripts for this issue. We greatly appreciate their help and contributions maintaining the quality of the journal:

- Alain Gilles FOKA TAGNE, University of Dschang, Cameroon.

- Hocine IFOURAH, University of Bejaia, Algeria

- Faten CHIBANI, University of Gabes, Tunisia

- Imen ABDENNADHER, University of Sfax, Tunisia

- Hicham SBAI, University Chouaib Doukkali, Al Jadida, Morocco

- Azzouz ZOUAOUI, U. Saudi Arabia

- Romuald TEMOMO WAMBA, University of Dschang, Cameroon

- Pr. Vincent MEYER, University of Nice, France

- Rabeb RIAHI, University of Gabes, Tunisia

- Saadou ABOUBACAR BARMOU, University of Niger

- Babarindé René ADEROMOU, university du Benin, special thanks !

We apologize for the delay in publishing this issue due to the overload of our volunteers.

Journal of Academic Finance is recruiting reviewers for the journal. We welcome you to join us.

\section{Remerciements aux réviseurs}

Journal of Academic Finance tient à remercier les personnes suivantes pour leur aide dans l'examen par les pairs des manuscrits pour ce numéro. Nous apprécions grandement leur aide et leurs contributions pour maintenir la qualité de la revue :

- Alain Gilles FOKA TAGNE, Université de Dschang, Cameroun.

- Hocine IFOURAH, Université de Bejaia, Algerie

- Faten CHIBANI, Université de Gabes, Tunisie

- Imen ABDENNADHER, University de Sfax, Tunisie

- Hicham SBAI, Université Chouaib Doukkali, Al Jadida, Maroc

- Azzouz ZOUAOUI, U. Arabie Seoudite

- Romuald TEMOMO WAMBA, Université de Dschang, Cameroun

- Pr. Vincent MEYER, Université de Nice, France

- Rabeb RIAHI, Université de Gabes, Tunisie

- Saadou ABOUBACAR BARMOU, Université du Niger

- Babarindé René ADEROMOU, université du Benin, Remerciements appuyés !

Nous présentons toutes nos excuses pour le retard de publication de ce numéro dû à la surcharge de travail de nos bénévoles.

Journal of Academic Finance recrute des réviseurs pour la revue. Nous vous invitons à nous rejoindre.

Editor-in-chief / Le rédacteur en chef

Pr. Jamel Eddine HENCHIRI 